Anita Pavić Pintarić ${ }^{*}$

Universität Zadar

\title{
DEUTSCHE UND KROATISCHE PHRASEME ZUM AUSDRUCK DES RAUMES
}

\section{EINLEITUNG}

Raum und Sprache erwecken zunehmend das Interesse der Sprachwissenschaft, wobei vorwiegend Raumausdrücke oder die Lexikalisierung der Raumkonzepte in unterschiedlichen Sprachen untersucht werden (vgl. Brala-Vukanović, Rubinić 2011: 22). Wie Brdar, Omazić et al. (2011: vii) hervorheben, finden alle menschlichen Aktivitäten in Raum und Zeit statt, was auch in der Sprache zum Ausdruck gebracht wird. Nach Gaines (2006: 173) ist Raum der Hintergrund für die Wahrnehmung der Welt, wobei dieser Hintergrund $u$. a. durch die Entfernung zwischen Objekten mitkonstituiert wird. Laut Wingender (1995: 80-83) wird Räumlichkeit zusammen mit Zeitlichkeit nach bestimmten Punkten sprachlich strukturiert: Menschen setzen Fixpunkte in Raum und Zeit an, die in der Sprache durch Deiktika ausgedrückt werden, wobei deiktische Zeichen kotext- und kontextgebunden sind ${ }^{1}$; Räumlichkeit kann topologisch erfasst werden, indem für eine Entität eine charakteristische Raumregion bestimmt werden kann, und Raum wird nach räumlichen Verhältnissen strukturiert (Richtung Kopf-Fuß oder Himmel-Erde und vorne-hinten oder auf-ab), wobei auch Entfernungen unterschieden werden können. Objekte werden in den Raum gestellt, und die Raumbeziehungen erfassen die Lage, Richtung und Ausdehnung (Šarić 2014: 25). Ortner (2013: 344) bietet einen Überblick zu raumlinguistischen Untersuchungen, die sich auf Formen von Raumreferenz beziehen und hebt dabei die Lokalisierung (Positionierung, Direktionalisierung) und die Dimensionierung (zwei- oder dreidimensionale Eigenschaften oder Typen von Körpern) mit ihren Unterformen hervor.

Laut Opašić (2011: 65) wird Raum als abstrakte Kategorie mit unterschiedlichen sprachlichen Einheiten wie Adverbien, Verben oder Pronomen aber auch mit Phrasemen ausgedrückt. Wunderlich (1985: 67) behauptet, Gestalt, Größe, Lage und charakteristische Bewegungen der Gegenstände seien Kriterien für ihre Identifizierung und Kategorisierung in einem Raum. Dazu hebt er Richtungen hervor, die adverbial benannt werden: oben und unten, vorne und hinten, rechts und links (ebd. 68).

anita.pintaric@unizd.hr

1 Huffschmid/Wildner (2009: 3) heben das Durchdringen von Kategorien wie Raum und Diskurs hervor: „Raum kann nicht ohne seine diskursive Konfiguration gedacht werden [...]. Diskurs wiederum entfaltet sich nicht im luftleeren, sondern in einem mehrdimensionalen Raum, im physischen wie auch im übertragenen Sinne“. Bauriedl (2007) bezeichnet den Raum in der Diskursforschung als Sozialgefüge, konkreten Ort, Diskurslandschaft sowie als Indikator eines Maßstabes. 
Wunderlich (ebd. 72) erwähnt „lexikalische Kategorien mit vorwiegend oder einem dominanten Anteil lokaler Ausdrücke“: Präpositionen (z. B. in, aus, vor, hinter, um), Adverbien (z. B. hier, da, dort, oben, unten), dimensionale Adjektive (z. B. hochniedrig, tief-flach, weit-eng), Positionsverben (z. B. liegen, stehen, wohnen), Bewegungsverben (z. B. gehen, fahren, steigen; legen, stellen, werfen) und Substantive (z. B. Spitze, Seite, Loch, Ecke).

Die Rolle, „die räumlichen Vorstellungen des Sprechers/Schreibers sprachlich zu vermitteln", übernehmen Lokaladverbien, die damit zu den Mitteln der lokalen Deixis gehören (Götze, Hess-Lüttich 2005: 290). In den Texten wird mit deiktischen Mitteln „auf Personen, Orts- und Zeitangaben der außersprachlichen Realität“ (ebd. 520) verwiesen, wobei deutlich ist, dass Adverbien nur in einer bestimmten Situation ihre Bedeutung realisieren, wenn die Rezipienten diese Situation nachvollziehen können.

Laut Duden (2009: 573) situieren Lokaladverbien ,ein Geschehen, ein Objekt o.̈̈. im Raum oder bezeichnen eine lokale Beziehung. Dabei unterscheidet man (statische) Ortsadverbien von (direktionalen) Richtungsadverbien“, wobei statische Lokaladverbien bspw. hier, da dort, unten, oben, links, rechts, außen oder innen und direktionale u. a. folgende sind: hin, her, dorthin, geradeaus, fort, weg (Duden 2009: 574).

In diesem Beitrag soll untersucht werden, wie das Konzept „Raum“ in der deutschen und kroatischen Sprache phraseologisch umgesetzt wird. Dabei wird „Raum“ laut Duden-Online ${ }^{2}$ als ein Nutz- oder Wohnraum, eine nicht eingegrenzte Ausdehnung, eine fest eingegrenzte Ausdehnung, ein für jemanden oder etwas zur Verfügung stehender Platz, Weltraum, ein geografisch oder politisch bestimmtes Gebiet und eine mathematische Menge von Koordinaten oder Elementen betrachtet. Im Kroatischen wird für „Raum“ noch die Bedeutung „Abstand“ im umgangssprachlichen Register angegeben. ${ }^{3}$

Im vorliegenden Beitrag werden folgende Fragen beantwortet: Wie wird auf Raum mit Phrasemen im Deutschen und Kroatischen referiert? Welche Konstituenten weisen auf den Raum hin? Welche räumlichen Dimensionen werden mit Phrasemen ausgedrückt?

\section{2 „RAUM“ IN DER PHRASEOLOGIE}

Sprachwissenschaftliche Studien zum Raum widmen ihre Aufmerksamkeit vorwiegend den Präpositionen, verbalen Präfixen, Verben und Substantiven mit räumlicher Bedeutung sowie Adverbien und Metaphern (vgl. z. B. Šarić 2014: 15). Daneben können aber auch Phraseme räumliche Bedeutungen ausdrücken. Laut Wunderlich (1985: 76) ist die räumliche Wahrnehmung eine reiche Quelle für Metaphern, wobei die Räumlichkeit ,auf die verschiedensten Bereiche des Wissens, des Bewußtseins, der Emotionen und Bewertungen ausgedehnt" wird. Er hebt weiterhin hervor, dass viele Metaphern als Idiome erstarrt sind und dass im Lexikon ,,unzählige idiomatische Ausdrücke mit ursprünglich lokalen Bedeutungen" (ebd. 77) gespeichert sind. Adverbialphrasen, mit denen Bewegung bezeichnet wird, sind u. a. „kreuz und quer“, „um herum“(Wildgen 2008: 109).

2 https://www.duden.de/rechtschreibung/Raum [02.10.2018].

3 http://hjp.znanje.hr/index.php?show=search [02.10.2018]. 
Phraseologische Untersuchungen widmen sich zunehmend dem Raum vor allem aus areal- (vgl. z. B. Piirainen, Piirainen 2002, Korhonen, Mieder et al. 2010) und kognitivlinguistischer Perspektive. So wird BehäLter in Bezug auf Emotionen (vgl. z. B. Kövecses 1986: 14 in Bezug auf Ärger) und auf Vorstellungsshemata in der Phraseologie (vgl. z. B. Parizoska, Novoselec 2010) untersucht. Im politischen Diskurs kommen konzeptuelle Metaphern in Bezug auf den Raum wie SUCESS IS VERTICAL MOVEMENT, LOVE IS A JOURNEY, LIFE IS A JOURNEY, PROGRESS IS FORWARD MOTION vor (vgl. z. B. Omazić 2015). Dobrovolskij und Piirainen (2009: 26) untersuchen u. a. die Typologie der Motivaton von Idiomen und nennen unter Idiomen mit ähnlichen Konstituenten und unterschiedlicher Bedeutung in mehreren Sprachen das Phrasem in den/seinen eigenen vier Wänden, dessen Slot ABGeSCHLOSSENER, ISOLIERTER RAum ist. Weiterhin werden Metaphern der Orientierung im Raum mit Ausgangskonzepten OBEN und unTEN genannt, die mit dem Beispiel im siebten Himmel sein erklärt werden, wobei „die räumliche Höhe mit GlüCKSEMPfindung, die Tiefe mit unglüCKLichem Gemütszustand gleichgesetzt werden" (Dobrovol'skij, Piirainen 2009: 39). Jachnow (2005: 247) untersuchte die Graduierungsphraseologie an einem bosnischen/kroatischen/serbischen Korpus und nannte Räumlichkeit als große Entfernung (dokle oko dopre/seže, iza devet gora, na kraju svijeta), räumliche Totalität (od glave do pete, na svakom koraku) und Bewegung im Raum (širi se kao vatra).

Raum als selbständiges Thema ist selten Gegenstand der phraseologischen Untersuchungen. Opašić (2011: 65-72) analysierte Raum und Zeit in der kroatischen Phraseologie und ging davon aus, dass mit Phrasemen räumliche und zeitliche Beziehungen ausgedrückt werden können. Sie untersuchte die Bedeutung der Phraseme sowie das phraseologische Bild. Phraseme mit der räumlichen Bedeutung werden in drei Gruppen eingeteilt: Ausdehnung (,rasprostranjenost"), große Entfernung (,velika udaljenost“) und kleine Entfernung (,mala udaljenost“). Dabei kann sich die Ausdehnung darauf beziehen, dass etwas überall (z. B. na sve četiri strane svijeta) oder an manchen Stellen (ovdje-ondje) zu finden ist. Bei diesen Phrasemen werden Raum, Bewegungsrichtung oder Bewegungsweise im Raum mit der bildlichen Konstituente dargestellt: Die kleine Entfernung wird mit dem Lexem Schritt (na dva, tri koraka do koga, čega) und die große Entfernung mit dem Lexem Welt (na kraj svijeta) dargestellt. Ferner kann die große Entfernung als Ort und Bewegungsrichtung erfasst werden.

Kržišnik (2014: 377-378) analysiert den Ausdruck von Raum in der slowenischen Phraseologie, wobei die meisten Phraseme eine somatische Konstituente haben. Sie unterscheidet Phraseme für den Ausdruck des eingegrenzten (z. B. Bezeichnungen für Planeten, Ozeane, Kontinente) und nicht eingegrenzten (z. B. brez konca in kraja, kamor oči nesejo/sežejo) Raumes sowie der räumlichen Dimensionen (z. B. Lage in Raum und Richtung).

\section{VORGEHEN UND KORPUSDARSTELLUNG}

Im Folgenden soll zunächst bestimmt werden, welche Dimensionen zur Bezeichnung des Raumes eruiert werden können. Im Anschluss sollen Phraseme zum Ausdruck räumlicher Dimensionen, die deutschen und kroatischen einsprachigen 
Wörterbüchern (Duden Redewendungen 2008, Duden-Online / Hrvatski jezični portal, Hrvatski frazeološki rječnik 2014) entnommen worden sind, eingehender untersucht werden. Zur Bestimmung der Dimensionen wurde nach Definitionen des Wortes „Raum“ in den oben genannten lexikografischen Quellen gesucht, die auch im Sachwörterbuch von Franz Dornseiff (2004) überprüft wurden. Die Sichtung verschiedener Einträge verdeutlicht, dass „Raum“ (un-)eingegrenzt und unter bestimmten Aspekten (geografisch, politisch, mathematisch usw.) definiert werden kann. Nach Dornseiff (2004: 31-46) wurden folgende Dimensionen für den „Raum“ bestimmt: Standort, Weite; Überall; Entfertntsein; Nähe; senkrecht, waagerecht; kreuzen; außen, innen; Umgrenzung; hinten, Zentrum, links, rechts; oben und unten. Für die Analyse wurden Phraseme mit den oben bestimmten Dimensionen als Konstituenten herangezogen sowie Phraseme, deren Bedeutung sich auf die festgestellten Dimensionen beziehen.

Das phraseologische Korpus verweist auf folgende Dimensionen: Weite; überall; Entfertntsein - Nähe und Standort. Daneben werden Phraseme nach den morphosyntaktischen und semantischen Merkmalen laut Burger (2015) beschrieben.

\section{ANALYSE}

In der Analyse wurden Dimensionen einbezogen, die nach lexikografischen Quellen und nach der Einteilung von Dornseiff (2004: 31-46) bestimmt wurden: „Weite“; ,Entferntsein - Nähe“; „Standort“ und ,überall“. Diese Dimensionen umfassen den Raum von seiner großen Ausdehnung und keiner Eingrenzung bis zu bestimmten Standorten.

\subsection{Dimension „Weite“}

Die Dimension „Weite“ bezieht sich auf einen weiten Raum und eine weite Fläche, auf Ferne, aber auch auf Größe, Umfang eines Körpers, Durchmessen eines Hohlraumes, einer Öffnung o. Ä. ${ }^{4}$ Diese Dimension umfasst Phraseme, die in ihrer Bedeutung Weite und den freien, offenenen Raum beinhalten. Diejenigen Phraseme, die eine große Entfernung bezeichnen, werden der Dimension „Entferntsein“ als Opposition zu der Dimension „Nähe“ zugeordnet. Die „Weite“ bezeichnen Phraseme, die im Deutschen Konstituenten wie das Freie $e^{5}$ und den Himmel enthalten, die selbst einen großen offenen Raum darstellen. Diese Konstituenten befinden sich in folgenden teilidiomatischen Phrasemen: im adverbialen Phrasem unter freiem Himmel sowie im verbalen Phrasem ins Freie gehen (Dornseiff, 2004: 33) und in dem adverbialen Phrasem in freier Wildbahn (,in der freien Natur", DRW). In DRW weist auch das idiomatische adverbiale Phrasem vor die Tür (,ins Freie“) auf die „Weite“ hin.

Im Kroatischen ist laut HFR ebenfalls die Konstituente Himmel in den adverbialen idiomatischen Phrasemen pod vedrim nebom (unter heiterem Himmel - „draußen,

4 https://www.duden.de/rechtschreibung/Weite [02.10.2018].

5 Das „Freie“ als Konstituente bezieht sich auf etwas, was draußen, in einem offenen Raum besteht und zeigt Überlappung mit der Dimension „außen“, die bei Dornseiff (2004: 37) genannt wird. Da werden adverbiale Phraseme im Freien, am Busen der Natur und unter freiem Himmel genannt. 
im Offenen“) und pod kapom nebeskom (unter der Himmelskappe - „in der ganzen Welt, auf der ganzen Erde") sowie nebu pod oblake (dem Himmel unter den Wolken - „auf die große Höhe fliegen, hochgehen“) enthalten. Zur Dimension „Weite“ werden folgende teilidiomatische Phraseme mit der Konstituente Welt zugeordnet: das verbale Phrasem vidjeti (proći) svijeta (Welt sehen/passieren - „durch viele Orte reisen") und das substantivische Phrasem bijeli svijet ${ }^{6}$ (die weiße Welt - „die ganze Welt mit guten und schlechten Eigenschaften"). Im Gespräch, wenn man feststellt, dass beide Kommunikationspartner die gleichen Personen kennen oder dass eine Nachricht schnell verbreitet wird, wird das Phrasem kako je svijet malen / die Welt ist klein als Kommentar verwendet.

\subsection{Dimension, „überall““}

Die Dimension ,überall“ bezieht sich u. a. auf Ausdehnung „an jeder Stelle, an jedem Ort $^{\text {" }}$ und wird im Deutschen mit Konstituenten Ecken und Enden, Seiten, Schritt und Tritt, nah und fern, Land, Weg und Steg und Welt signalisiert. Diese Konstituenten weisen selbst räumliche Dimensionen und Bewegung aus unterschiedlichen Richtungen auf. Mit folgenden idiomatischen adverbialen Phrasemen wird also die Dimension „überall“" ausgedrückt: an allen Ecken und Enden, von allen Seiten, auf Schritt und Tritt, von nah und fern (Dornseiff 2004: 34), landaus, landein, auf Weg und Steg, weit und breit, in aller Welt, in alle [vier] Winde, wo jmd. geht und steht (DRW), wobei ebenfalls folgende teilidiomatische adverbiale Phraseme belegt sind: aus aller Welt, in alle Welt und landauf, landab (DRW). Die Bewegung in alle Richtungen bezeichnet das idiomatische adverbiale Phrasem kreuz und quer (DRW).

Im Kroatischen bezeichnen laut HFR idiomatische adverbiale Phraseme na sve strane (,auf alle Seiten"), sa svih strana (,von allen Seiten“), na sve četiri strane svijeta (,in alle vier Himmelsrichtungen“), i lijevo i desno (pokazati što i sl.) (,,links und rechts zeigen“), na svakom koraku (,auf jedem Schritt“), na svakom uglu (ćošku) (,,an jeder Ecke“) die Dimension „überall“, wobei die Konstituenten Seiten, Ecke und Schritt eine Rolle spielen und Bewegungen aus unterschiedlichen Richtungen bezeichnen. Neben diesen haben laut HFR folgende adverbiale Phraseme die Bedeutung ,,in alle Richtungen": gore-dolje, amo-tamo, $<i>$ lijevo i desno, wobei die letzten zwei auch Unsicherheit, Zweideutigkeit und Zögerung bezeichnen können. Keine bestimmte Lage, kein bestimmter Ort wird mit den adverbialen Phrasemen überall und nirgends (DRW) und ni na nebu ni na zemlji (HFR), die mit ihren Konstituenten einen weiten Raum darstellen, ausgedrückt.

Teile des Raumes werden mit der Bedeutung „mancherorts“ erfasst, und zwar durch adverbiale Phraseme mit den Konstituenten da, dort und hier, deren Gebrauch als selbstständige Adverbien normalerweise von der Perspektive des Sprechers abhängt, was aber in den Phrasemen keine Rolle spielt: da und dort, hier/hie und dort/da (DRW), tu i tamo (HFR, „da und dort"), ovdje-ondje (HFR, „hier-dort").

6 http://hjp.znanje.hr/index.php?show=search [02.10.2018].

7 https://www.duden.de/rechtschreibung/ueberall [02.10.2018]. 


\subsection{Dimension „Enferntsein“ - „Nähe“}

Dimensionen „Entferntsein“ und „Nähe“ werden als Oppositionen im Raum dargestellt. „Entferntsein“ wird durch adverbiale idiomatische Phraseme mit den Konstituenten Berge, Reichweite und Ende der Welt, die auf die Entfernung hinweisen, ausgedrückt: außer Reichweite, über alle Berge (Dornseiff, 2004: 34) sowie so weit das Auge reicht, am Ende der Welt und bis ans Ende der Welt (DRW). Es gibt auch einige scherzhafte satzwertige Phraseme mit Zoonymen, die einen sehr abgelegenen Ort bezeichnen: wo sich die Füchse gute Nacht sagen (Dornseiff, 2004: 34), wo sich Fuchs und Hase gute Nacht sagen, wo die Hunde mit dem Schwanz bellen (DRW). Diese Bedeutung drücken noch das umgangssprachliche Phrasem hier ist die Welt [wie] mit Brettern vernagelt (DRW) aus, das einen abgelegenen und langweiligen Ort bezeichnet und das als derb bezeichnete Phrasem am/an den Arsch der Welt (DRW).

Im Kroatischen wird „Entferntsein“ laut HFR mittels der adverbialen idiomatischen Phraseme mit den Konstituenten Berge, Ende der Welt und den Körperteilen Hand und Auge ausgedrückt: preko (iza) devet (sedam) gora (brda, mora) (,,̈̈ber/ hinter neun/sieben Berge/Meere“), na kraju svijeta (,,am Ende der Welt"), na kraj svijeta (ići, poći $i$ sl.) (,ans Ende der Welt gehen“), preko brda i dolina (,über Berge und Täler"), $<n a>$ tri dana jahanja (,drei Tage reiten“), dokle oko seže (dopire $i$ sl.) (,so weit das Auge reicht“), izvan dohvata koga, čega (,,außer jemandes Reichweite"), (nalaziti se) izvan ruke (,sich außer Hand befinden“). Es bestehen wie im Deutschen umgangssprachliche, scherzhafte idiomatische adverbiale Phraseme und ein satzwertiges Phrasem in der Bedeutung eines sehr weit abgelegenen Ortes: Bogu iza leđa (za leđima) („dem Gott hinter den Rücken“), Bogu iza nogu („,dem Gott hinter den Beinen“) und gdje je Bog rekao laku noć (,wo Gott Gute Nacht sagte“).

Zur Bezeichnung der "Nähe“ werden die Konstituenten dicht, Hörweite und Reichweite in folgenden deutschen adverbialen idiomatischen Phrasemen sowie Nase und Fersen in verbalen Phrasemen verwendet: dicht bei dicht, in Hörweite, in Reichweite (Dornseiff, 2004: 35), jemandem auf den Fersen sein/bleiben, vor jmds. Nase, etwas vor der Nase haben (DRW).

Im Kroatischen sind Konstituenten die Körperteile Hand, Nase, Fersen und Schritte, die in folgenden idiomatischen verbalen Phrasemen mit den Verben sein oder haben verbunden werden: (tu je) pod nosom (,es ist hier unter der Nase"), biti kome za petama (,jemandem hinter den Fersen sein“), (nema ni) dva koraka (odavde) ${ }^{10}$ (,es gibt sogar nicht zwei Schritte von hier"), biti na korak od (do) čega (,,auf einem Schritt von etwas sein“) (HFR), na dohvat ruke (,,in der Reichweite des Armes sein“) (HFR), biti (naći se) pri ruci komu (,jemandem bei der Hand sein/sich befinden") (HFR).

\subsection{Dimension ,Standort“"}

8 http://hjp.znanje.hr/index.php?show=search_by_id\&id=eFxmURg\%3D [02.10.2018].

9 http://hjp.znanje.hr/index.php?show=search_by_id\&id=eV51XRU\%3D [02.10.2018].

10 http://hjp.znanje.hr/index.php?show=search [02.10.2018]. 
Im Raum können die Standorte spezifiziert werden, und Gegenstände können sich ebenfalls auf mehrere Orte in einem Raum beziehen. Der bestimmte Standort wird als „Ort“ oder „Punkt" bezeichnet, an dem jemand oder etwas steht. Weitere Bedeutungen des Wortes „Standort“ beziehen sich auf militärische Dienststellen, Einrichtungen und Anlagen sowie auf den geografischen Ort oder Raum, wo bestimmte wirtschaftliche Aktivitäten stattfinden. ${ }^{11}$

Der „Standort“ wird in beiden Sprachen mit den raumbezeichnenden Konstituenten Ort, Stelle, Wände aber auch mit Körperteilen Augen, Nase, Fuß signalisiert. Diese Dimension drücken idiomatische adverbiale Phraseme an Ort und Stelle, in seinen vier Wänden sowie na licu mjesta (,am Gesicht des Ortes") und između (vlastita) četiri zida (,zwischen den eigenen vier Wänden“) aus. Das Phrasem unter vier Wänden verwendet man in beiden Sprachen, wenn man sein eigenes Zuhause bezeichnet, in dem man sich wohl fühlt. Dabei kann dieses Phrasem in beiden Sprachen auch die Dimension „innen“ bezeichnen (Dornseiff 2004: 37). Im Kroatischen gehören zu dieser Dimension noch idiomatische substantivische Phraseme krov nad glavom (Dach über dem Kopf, „eigenes Zuhause“) und luka spasa (Hafen der Rettung, „Zufluchtsort", HFR).

Geografische Räume, die mit Phrasemen in beiden Sprachen bezeichnet werden, sind: die Alte Welt - Stari svijet („Europa“), die Neue Welt - Novi svijet („Amerika“), die Dritte Welt - Zemlje trećeg svijeta („Länder der dritten Welt“), das Heilige Land - Sveta zemlja (biblisch), das Land der aufgehenden Sonne - Zemlja izlazećeg sunca („Japan“), der Blaue Planet - Plavi planet („Erde“), der Rote Planet - Crveni planet (,Mars"). Im Deutschen ist noch die Bezeichnung die Vierte Welt (,die ärmsten Entwicklungsländer", DRW) gängig.

Neben genau bestimmten geografischen Bezeichnungen können mit Phrasemen auch Orte bezeichnet werden, die zwar keinen genauen Standort bezeichnen, aber deren entweder positive oder negative Werte hervorheben. Als positiv bewertet gelten Orte, wo alle glücklich und zufrieden sind, was in folgenden idiomatischen substantivischen Phraseme ausgedrückt wird: Insel der Glückseligen, das Land, wo Milch und Honig fließt (DRW), teče med i mlijeko < gdje> („Honig und Milch fließt“", „alles ist im Überfluss vorhanden, es kann nicht besser sein /über ein Gebiet/ HFR) und mjesto pod suncem („Platz unter der Sonne“, „günstige Lage, Platz für das Leben oder Arbeit“ HFR). Das Befinden am richtigen Ort zur bestimmten Zeit bezeichnen Phraseme mit den Konstituenten Stelle, Ort und Platz: zur Stelle sein, jmds. Platz ist bei jmdm./an einem bestimmten Ort (DRW).

Negativ bewertete Orte erfassen bspw. substantivische Phraseme Sodom und Gomorrha und Sodoma i Gomora sowie satzwertige, als umgangssprachlich und scherzhaft markierte Phraseme (DRW): ein heißes, teures o. Ä. Pflaster sein, auf der falschen Beerdigung sein, da/dort möchte ich nicht begraben sein, da möchte ich nicht tot überm Gartenzaun hängen.

Man kann sich im Raum aufhalten oder den Raum verlassen. Den Aufenthalt oder

11 https://www.duden.de/rechtschreibung/Standort. [02.10.2018]. 
das Verweilen im Raum bezeichnen laut DRW deutsche verbale Phraseme wie bei jmdm., in etw. aus und ein/ein und aus gehen und die Gegend unsicher machen. Den Raum kann man verlassen oder aus ihm ausgewiesen werden. Im Deutschen wird dies mit verbalen Phrasemen wie z. B. jmdn. achtkantig/vierkantig hinauswerfen/rausschmeißen und jmdm. die Tür weisen ausgedrückt, wobei im Kroatischen das verbale Phrasem pokazati kome vrata (,jemandem die Tür zeigen“, HFR) mit der Konstituente Tür vorhanden ist.

\section{SCHLUSSFOLGERUNG}

Neben lokalen Ausdrücken, die vor allem Adverbien, Präpositionen, Verben und Substantive umfassen, stellt sich nach der vorliegenden Untersuchung heraus, dass auch Phraseme zur Bezeichnung des Raumes, der Ausdehnung, der Entfernung und des bestimmten Standortes verwendet werden. Räumliche Dimensionen, auf die in der deutschen und kroatischen Sprache mit Phrasemen referiert wird, sind: „Weite“; ,überall“; „Entfertntsein - Nähe“ und „Standort“. Die Dimension „Weite“ bezieht sich auf den offenen, freien Raum, auf die ganze Welt, auf die Natur und auf viele Orte, wobei in beiden Sprachen idiomatische adverbiale Phraseme mit den Konstituenten das Freie, Himmel und Welt auf diese Dimension referieren.

Die Dimension ,überall“", deren Bedeutung keinen bestimmten Ort und keine bestimmte Lage umschreibt, sondern alle Richtungen und Distanzen, wird mit den Konstituenten Ecken, Seiten, Schritt, Weg, Welt, oben - unten, aus - ein, auf-ab, links und rechts signalisiert. Die Phraseme von allen Seiten/sa svih strana, da und dort/tu i tamo, hier/hie und da/ovdje - ondje werden in beiden Sprachen verwendet.

Die Dimensionen „Entferntsein“ und „Nähe“ werden mit den meisten Phrasemen ausgedrückt. Auf die „große Entfernung“" wird mit den Konstituenten Reichweite, Berge, Ende der Welt und auf die „Nähe“ zusätzlich noch mit Nase, Fersen und Schritte in adverbialen Phrasemen referiert, wobei für sehr entfernte, abgelegene und unwichtige Orte satzwertige Phraseme mit Zoonymen im Deutschen und mit der Konstituente Gott im Kroatischen verwendet werden. Entsprechungen finden sich unter folgenden Phrasemen, die eine „große Entfernung“ bezeichnen: am Ende der Welt, ans Ende der Welt / na kraju svijeta, na kraj svijeta, die Welt ist klein / kako je svijet malen; über alle Berge / preko (iza) sedam (devet) gora (brda, mora); so weit das Auge reicht / dokle oko seže (dopire). Bei der Bezeichnung der "Nähe“ stehen folgende Phraseme in beiden Sprachen zur Verfügung: in Reichweite / biti na dohvat ruke; etwas vor der Nase haben / (tu je) pod nosom; jmdm. auf den Fersen sein, beleiben / biti kome za petama.

Unter „Standorten“ werden u. a. das Zuhause, der Zufluchtsort, geografische Standorte sowie positiv und negativ bewertete Orte aus der Perspektive des Sprechers verstanden. Es lassen sich folgende ausmachen: in seinen vier Wänden / između (vlastita) četiri zida, die Alte Welt /Stari svijet; die Neue Welt / Novi svijet; das Heilige Land / Sveta zemlja; der Blaue Planet / Plavi planet; der Rote Planet / Crveni planet. Zur negativen Bewertung verfügt die deutsche Sprache über satzwertige umgangssprachliche und scherzhafte Phraseme, die Orte aus der Perspektive des Sprechers in der ersten Person Singular als gefährlich und nicht zum Leben geeignet bezeichnen. Im Kroatischen 
sind solche Phraseme nicht vorhanden.

Allgemein kann behauptet werden, dass beide Sprachen sehr ähnlich auf den Raum mit Phrasemen referieren. Entsprechungen sind nicht nur in der phraseologischen Versprachlichung der räumlichen Dimensionen auszumachen, sondern auch in den Phrasemen selbst aus morphosyntaktischer und semantischer Sicht, was ein Anlass zu weiteren raumlinguistischen Untersuchungen im Rahmen der Phraseologie sein kann.

\section{Literatur}

BRALA-VUKANOVIĆ, Maja/Nensi RUBINIĆ (2011) „Prostorni prijedlozi i prefksi u hrvatskome jeziku." Fluminensia 23/2, 21-37.

BRDAR, Mario/Marija OMAZIĆ/Višnja PAVIČIĆ TAKAČ/Tanja GRADEČAKERDELJIĆ/Gabrijela BULJAN (Hrsg.) (2010) Prostor $i$ vrijeme u jeziku: Jezik u prostoru i vremenu. Zagreb/Osijek: HDPL, Filozofski fakultet Sveučilišta Josipa Jurja Strossmayera.

BURGER, Harald (2015) Phraseologie: Eine Einführung am Beispiel des Deutschen. Berlin: Erich Schmidt.

DOBROVOL'SKIJ, Dmitrij/Elisabeth PIIRAINEN (2009) Zur Theorie der Phraseologie. Kognitive und kulturelle Aspekte. Tübingen: Stauffenburg.

DORNSEIFF, Franz (2004) Der deutsche Wortschatz nach Sachgruppen: Mit einer lexikographisch-historischen Einführung und einer ausführlichen Bibliographie zur Lexikographie und Onomasiologie. Berlin/New York: de Gruyter.

DUDEN Grammatik der deutschen Gegenwartssprache (2009). Mannheim: Bibliographisches Institut.

DUDEN (2008) Redewendungen 11 Wörterbuch der deutschen Idiomatik. Mannheim/ Leipzig/Wien/Zürich: Dudeverlag.

GAINES, Elliot (2006) „Communication and the Semiotics of Space.“ Journal of Creative Communications 1/2, 173-181.

GÖTZE, Lutz/Ernest W. B. HESS-LÜTTICH (2005) Grammatik der deutschen Sprache. Gütersloh/München: Bertelsmann Lexikon Institut.

JACHNOW, Helmut (2005) „Graduierungsphraseologie - Wesen und Funktion, dargestellt an einem bosnischen/kroatischen/serbischen Korpus.“ In: H. Jachnow/A. Kiklevič/N. Mečkovskaja/B. Norman/M. Wingender (Hrsg), Kognition, Sprache und phraseologische/parömiologische Graduierung. Wiesbaden: Harrassowitz Verlag, 154-296.

KORHONEN, Jarmo/Wolfgang MIEDER/Elisabeth PIIRAINEN/Rosa PIÑEL (2010) (Hrsg.) Phraseologie global - areal - regional. Akten der Konferenz EUROPHRAS 2008 vom 13.-16.8.2008 in Helsinki. Tübingen: Narr Francke Attempto.

KÖVECSES, Zoltán (1986) Metaphors of anger, pride, and love. A lexical approach to the structure of concepts. Amsterdam: Benjamins.

KRŽIŠNIK, Erika (2014) „Izražanje prostora v slovenski frazeologiji.“ Slavistična revija 62/3, 375-384.

MENAC, Antica/Željka FINK ARSOVSKI/Radomir VENTURIN (2014) Hrvatski frazeološki rječnik. Zagreb: Naklada Ljevak.

OMAZIĆ, Marija (2015) Phraseology through the looking glass. Osijek: Filozofski 
fakultet Sveučilišta Josipa Jurja Strossmayera.

OPAŠ́Ć, Maja (2011) „Prilog analizi kategorije vremena i prostora u hrvatskoj frazeologiji.“" Lahor 11, 65-87.

ORTNER, Heike (2013) „Die Online-Sphäre als Kommunikationsraum: Selbstverortung, Nähe und Distanz“. In: F. Cambi/W. Hackl (Hrsg.), Topographie und Raum in der deutschen Sprache und Literatur. Wien: Praesens, 338-354.

PARIZOSKA, Jelena/Zvonimir NOVOSELEC (2010) „Predodžbena shema SPREMNIKA u frazeologiji: prvi i drugi plan.“ In: M. Brdar/M. Omazić/V. Pavičić Takač/T. Gradečak-Erdeljić/G. Buljan (Hrsg.), Prostor $i$ vrijeme u jeziku: Jezik prostoru $i$ vremenu. Zagreb/Osijek: Hrvatsko društvo za primijenjenu lingvistiku/Filozofski Fakultet Sveučilišta Josipa Jurja Strossmayera, 57-67.

PIIRIANEN, Elisabeth/Ilpo Tapani PIIRAINEN (Hrsg.) (2002) Phraseologie in Raum und Zeit. Akten der 10. Tagung des Westfälischen Arbeitskreises „Phraseologiel Parömiologie“ (Münster 2001). Hohengehren: Schneider Verlag.

ŠARIĆ, Ljiljana (2014) Prostor u jeziku i metafora. Kognitivnolingvističke studije o prefiksima i prijedlozima. Zagreb: Jesenski i Turk.

WILDGEN, Wolfgang (2008) Kognitive Grammatik: Klassische Paradigmen und neue Perspektiven. Berlin/New York: Walter de Gruyter.

WINGENDER, Monika (1995) Temporalität und ihre Repräsentation im Lexikon des Russischen. Wiesbaden: Harrassowitz Verlag.

WUNDERLICH, Dieter (1985) „Raum, Zeit und das Lexikon.“ In: H. Schweizer (Hrsg.), Sprache und Raum. Psychologische und linguistische Aspekte der Aneignung und Verarbeitung von Räumlichkeit. Stuttgart: J. B. Metzlersehe Verlagsbuchhandlung, 66-89.

\section{Internetquellen}

BAURIEDL, Sybille (2007) „Räume lesen lernen: Methoden zur Raumanalyse in der Diskursforschung." Forum Qualitative Sozialforschung, FQS 8 (2), Art. 13. http:// www.qualitative-research.net/index.php/fqs/rt/printerFriendly/236/523 [07.10.2018].

Duden-Online. https://www.duden.de/ [02.10.2018].

Hrvatski jezični portal. http://hjp.znanje.hr/ [02.10.2018].

HUFFSCHMID, Anne/Kathrin WiLDNER (2009) „Räume sprechen, Diskurse verorten? Überlegungen zu einer transdisziplinären Ethnografie." Forum Qualitative Sozialforschung 10 (3). http://www.qualitative-research.net/index.php/fqs/article/ view/1224 [07.10.2018].

\section{Zusammenfassung \\ DEUTSCHE UND KROATISCHE PHRASEME ZUM AUSDRUCK DES RAUMES}

In diesem Beitrag werden Phraseme zur Bezeichnung des Raumes in der deutschen und kroatischen Sprache untersucht. Es wird von räumlichen Dimensionen ausgegangen, die anhand der grammatischen, semantischen und raumlinguistischen 
Untersuchungen bestimmt wurden. Phraseme wurden in einsprachigen allgemeinen und phraseologischen Wörterbüchern gesucht, wobei ihre morphosyntaktische und semantische Klassifikation bei der Analyse in Betracht gezogen wurden.

Die Analyse erwies folgende räumliche Dimensionen, die in beiden Sprachen mit Phrasemen ausgedrückt werden können: „Weite“, „überall“, „Entfernt sein“ - „Nähe“ und „Standort“. Für die Dimension „Weite“ werden in beiden Sprachen idiomatische adverbiale Phraseme mit Konstituenten das Freie, Himmel und Welt verwendet. Die Dimension „überall“ weist auf alle Richtungen und Distanzen und wird mit den Konstituenten Ecken, Seiten, Schritt, Weg, Welt, oben - unten, aus - ein, auf - ab, links und rechts signalisiert. In der Dimension „Entferntsein“ - „Nähe“ signalisieren Konstituenten Reichweite, Berge und Ende der Welt eine „große Entfernung“, während auf die „Nähe" noch mit Körperteilen Nase, Fersen sowie mit Schritten hingewiesen wird. Die letzte Dimension „Standort“ umfasst u. a. das Zuhause, den Zufluchtsort" sowie geografische Standorte und zeichnet sich durch positive und negative Bewertung aus.

Schlüsselwörter: Phraseme, Raum, räumliche Dimensionen, Deutsch, Kroatisch

\section{Abstract}

\section{GERMAN AND CROATIAN PHRASEMES AS EXPRESSIONS OF SPACE}

This paper investigates phrasemes used for expressing space in the German and Croatian languages. Dimensions of space, which are determined according to grammatical, semantic and spatio-linguistic investigations, are considered. The phrasemes were collected from general and phraseological dictionaries in each language. Morphosyntactic and semantic classifications were used in the analysis.

The analysis shows the following spatial dimensions, which can be expressed with phrasemes in both languages: "width", "everywhere", "distance", "vicinity" and "location". The "width" dimension can be represented with idiomatic adverbial phrasemes containing the constituents the open, sky and world. In the "everywhere" dimension, all directions and distances are signalled with the constituents corner, side, pace, way, world, up - down, from - in, from - to, left and right. The constituents range, mounts and the end of the world are used to refer to large distances within the "distance" dimension, while nose, heel and pace belong to the "vicinity" dimension. The last dimension, "location", includes home, shelter and also geographical sites and distinguishes between positive and negative values.

Key words: phrasemes, space, spatial dimensions, German, Croatian 


\section{Povzetek}

\section{NEMŠKI IN HRVAŠKI FRAZEMI ZA IZRAŽANJE PROSTORA}

Prispevek predstavlja raziskavo frazemov za izražanje prostora v nemškem in hrvaškem jeziku.Osnova za raziskavo so prostorske dimenzije, kot jih opredeljujejo slovnične, pomenske in prostorskolingvistične raziskave. Vir za analizirane frazeme so bili enojezični splošni in frazeološki slovarji, pri čemer je bila pri analizi upoštevana njihova morfosintaktična in semantična klasifikacija.

Analiza je razkrila naslednje dimenzije prostora, ki se v obeh jezikih izražajo s frazemi: „daljava“, „povsod“, „oddaljenost - bližina“ ter „nahajanje“. Za dimenzijo „daljava“" se v obeh jezikih uporabljajo idiomatski prislovni frazemi s sestavinami $n a$ prostem, nebo in svet. Dimenzija ,povsod“ kaže na vse smeri in distance in je signalizirana s sestavinami koti, strani, korak, pot, svet, zgoraj-spodaj, ven - noter, navzgor - navzdol, levo in desno. V dimenziji „oddaljenost - bližina“ signalizirajo sestavine doseg, gore in konec sveta „veliko oddaljenost“, medtem ko na „bližino“ kažejo deli telesa: nos, pete kot tudi koraki. Zadnja dimenzija, „nahajanje“, obsega med drugim dom, zatočišče kot tudi geografske lokacije, zanjo pa je značilna pozitivna oz. negativna konotacija.

Ključne besede: frazemi, prostor, prostorske dimenzije, nemščina, hrvaščina 